the results of reference 1 required by these considerations is easily made. The matrix element appropriate to a collision in which the $(K, p)$ system in a state $\psi_{n, l}$ is changed to a state $\psi_{n, l-1}$, which can be the products of the $K-p$ interaction, is

$$
\left\langle U_{L+1}{ }^{*} \psi_{n, l-1}{ }^{*}|H| U_{2} \psi_{n, l}\right\rangle,
$$

where the $U$ are the plane wave functions representing the relative $(K, p)$ atom and proton coordinates and $H$, the Hamiltonian, will be equal to $\left(e^{2} /\left|R-r_{p}\right|\right)-\left(e^{2} /\left|R-r_{k}\right|\right)$, where $R$ is the vector coordinate of the colliding proton and $r_{p}$ and $r_{k}$ are the coordinates of the proton and $K$ meson in the atom. For $|R|>|r|$ a multipole expansion of $H$ can be made. Setting $R=a_{0}$ as in reference 1 , the matrix element can be rewritten as

$$
\left\langle V_{L+1}{ }^{*}\left(a_{0}\right) \mid V_{L}\left(a_{0}\right)\right\rangle\left\langle\psi_{n, l-1}{ }^{*}\left|H^{\prime}\right| \psi_{n, l}\right\rangle \text {, }
$$

where, with appropriate averaging of geometric factors, the second term is precisely that evaluated by Day et al. In the first factor $V$ represents the radial part of the wave functions $U$, and the square of this term has a value of $1 / 5$ for $S$ to $P$ transitions which are the most favorable. Changes of more than one unit of angular momentum are much more strongly forbidden. These corrections modify the conclusions of reference 1 concerning the $n=6$ state in the following way. The depopulation of the $P$ level in any collision is essentially unaffected but the reshuffling of other states is much reduced and their direct depopulation is largely forbidden. This greatly reduces the transfer into the $P$ level and the average atomic lifetime is considerably increased, enhancing the importance of radiative transitions. Calculations of the same kind as reported in reference 1 lead then to the result that about $20 \%$ of atoms in a $n=6$ state reach the $2 P$ state instead of the $1.4 \%$ stated in reference 1.

The uncertainties involved in the estimates made in this note, and also in reference 1 , are quite large, and the conclusions reached in these calculations are not presented with the intention of establishing that $P$-wave capture is large, or that the Stark effect is unimportant. But we believe that these results do indicate the necessity of a more detailed examination of the problem.

\footnotetext{
${ }^{1}$ Day, Snow, and Sucher, Phys. Rev. Letters $\underline{3}, 61$ (1959).

${ }^{2}$ L. B. Okun' and I. A. Pomeranchuk, J. Exptl. Theoret. Phys. U.S.S.R. 34 , 997 (1958) [translation: Soviet Phys. JETP $\underline{34}, 688$ (1958)].
}

\title{
GRAVITATIONAL RED-SHIFT IN NUCLEAR RESONANCE
}

\author{
R. V. Pound and G. A. Rebka, Jr. \\ Lyman Laboratory of Physics, Harvard University, Cambridge, Massachusetts
} (Received October 15, 1959)

It is widely considered desirable to check experimentally the view that the frequencies of electromagnetic spectral lines are sensitive to the gravitational potential at the position of the emitting system. The several theories of relativity predict the frequency to be proportional to the gravitational potential. Experiments are proposed to observe the timekeeping of a "clock" based on an atomic or molecular transition, when held aloft in a rocket-launched satellite, relative to a similar one kept on the ground. The frequency $\nu_{h}$ and thus the timekeeping at height $h$ is related to that at the earth's surface $\nu_{0}$ according to

$$
\begin{array}{rl}
\Delta \nu_{h}=\nu_{h}-\nu_{0}=\nu_{0} & g h / c^{2}(1+h / R) \\
& \approx \nu_{0} h \times\left(1.09 \times 10^{-18}\right),
\end{array}
$$

where $R$ is the radius of the earth and $h$ is the altitude measured in $\mathrm{cm}$. Very high accuracy is required of the clocks even with the altitudes available with artificial satellites. Although several ways of obtaining the necessary frequency stability look promising, it would be simpler if a way could be found to do the experiment between fixed terrestrial points. In particular, if an accuracy could be obtained allowing the measurement of the shift between points differing as little as one to ten kilometers in altitude, the experiment could be performed between a mountain and a valley, in a mineshaft, or in a borehole.

Recently Mössbauer has discovered ${ }^{1}$ a new aspect of the emission and scattering of $\gamma$ rays by nuclei in solids. A certain fraction $f$ of $\gamma$ rays of the nuclei of a solid are emitted without 
individual nuclear recoil. Instead, the recoil momentum is delivered to the crystal lattice as a whole resulting in negligible Doppler shift. Such $\gamma$ rays are in resonance with nuclei similarly bound in a lattice and a similar fraction $f$ of the electromagnetic resonant cross section

$$
\sigma_{R}=2 \pi \lambda^{2}\left(\frac{2 I_{e}+1}{2 I_{g}+1}\right) \frac{1}{1+\alpha},
$$

where $I_{e}$ and $I_{g}$ are the spins of the emitting and the ground states, respectively, and $\alpha$ is the internal conversion coefficient, pertains to the scattering. Calculations based on the Debye model of lattice vibrations yield for $f$ at temperatures $T$ much less than the Debye temperature $\theta_{D}$

$$
f=\exp \left\{-\frac{3}{2} \frac{E_{\gamma}^{2}}{2 M c^{2} k \theta_{D}}\left[1+\frac{2}{3}\left(\frac{\pi T}{\theta_{D}}\right)^{2}\right]\right\},
$$

where $E_{\gamma}$ is the energy of the $\gamma$ ray, $M$ is the nuclear mass, and $k$ is Boltzmann's constant. The factor $\left(E_{\gamma}{ }^{2} / 2 M c^{2} k \theta_{D}\right)$ is the ratio of the recoil energy that would be taken up by the free nucleus to $k \theta_{D}$. For $\gamma$ rays much above the $129 \mathrm{kev}$ employed by Mössbauer the factor $f$ becomes very small even at absolute zero.

The most striking evidence for the existence of this effect is the observation that the attenuation of the 129-kev gamma rays of $\mathrm{Ir}^{191}$ in passing through an iridium absorber is reduced if the source is moved. The speed required to reduce the part of the attenuation caused by resonant scattering to one-half its maximum value was found to be approximately $1.5 \mathrm{~cm} / \mathrm{sec}$. From this a half-life of the excited state is derived to be $0.1 \mathrm{~m} \mu \mathrm{sec}$. Others have repeated this experiment, and extended it to helium temperatures. ${ }^{2,3}$ One other case is reported, ${ }^{3}$ that of $\mathrm{W}^{\mathbf{1 8 2}}$ wherein a half-life of $0.6 \mathrm{~m} \mu \mathrm{sec}$ is inferred by the Doppler width of the resonance. This is half the accepted lifetime as measured by delay coincidence techniques. It is not clear whether this discrepancy represents a limit of the technique or whether it is largely an instrumental problem, as the authors suggest, enhanced by the complex array of other $\gamma$ rays in the $\mathrm{Ta}^{182}$ source. Of course, as has been suggested, one should expect to see effects caused by hyperfine structure in these spectra when lifetimes are long enough to allow them to be important. All the effects discussed in connection with the directional correlation of cascade $\gamma$ rays should have an influence. For example, it would seem desirable to use a source that has a good chance of being in a normal lattice site and electronic state at the time of emission of the final $\gamma$ ray in question. One could have serious aftereffects from $\beta$ decays, from prior emission of high-energy $\gamma$ rays, or from electron captures as well as broadening from imperfections in the crystal lattice or short spin-lattice relaxation.

Even if the further development of the technique does not yield still narrower resonances, those already observed have fractional widths in frequency well below those of all the reference lines yet proposed for "atomic clocks." If the scattering is reduced to one-half its maximum by relative motion of the source and scatterer with velocity $v$, the $Q$, the ratio of the frequency to the full width at half-height of the resonance line being observed, is just $c / 2 v$. In the case of Mössbauer's experiment $Q$ is about $1 \times 10^{10}$ and in the case of $\mathrm{W}^{182}$ it is $7 \times 10^{10}$. In general $Q=1.10 E_{\gamma}(\mathrm{Mev}) \tau_{1 / 2}(\mathrm{~m} \mu \mathrm{sec}) \times 10^{12}$.

A measurement of the gravitational red shift could be performed by transmitting $\gamma$ rays from a source to a scatterer at an altitude different by $h$ and by observing what relative velocity yields maximum scattering. For the predicted shift to be a full half-width of the line, the altitude difference $h$ must be $h_{1 / 2}=\left[4.18 / E_{\gamma}(\mathrm{Mev}) \tau_{1 / 2}(\mathrm{~m} \mu \mathrm{sec})\right]$ $\mathrm{km}$. Thus, for the width reported for $\mathrm{W}^{182}, 66$ $\mathrm{km}$ difference of height would be required.

It is exciting to speculate about the possibilities opened up if cases of even less breadth can be found. For example, $\mathrm{Fe}^{57}$, for which $E_{\gamma}=0.0144$ Mev and $\tau_{1 / 2}=100 \mathrm{~m} \mu \mathrm{sec}$, would require only 2.9 $\mathrm{km}$ separation were it to yield its natural breadth. Another example might be $\mathrm{Zn}^{67}$ with an excited level at $0.093 \mathrm{Mev}$, of half-life $9400 \mathrm{~m} \mu \mathrm{sec}$. For this, if the natural breadth were obtained, $h_{1 / 2}$ would be 4.74 meters. This possibility represents a considerable extrapolation from present data. We are undertaking to examine these and other isotopes in various environments with the aim of selecting an isotope suitable for a gravitational experiment. Among other things equivalence of or absence of hyperfine structures in the sources and scatterers would be desirable.

Obviously one of the difficulties with large separations between source and scatterer arises from the inverse-square law of intensity. As a consequence of the participation of a large number of identical nuclei in an individual recoilfree scattering process, one anticipates the existence of intense Bragg diffraction from thin crystals. Thus one has the possibility of some 
degree of focusing with bent crystals. Furthermore one may use the Bragg reflection from thin crystals to separate the $\gamma$ rays emitted without recoil from all others. In this way irrelevant background $\gamma$ rays could be eliminated from the detector.

Total external reflection of low-energy $\gamma$ rays at grazing angles of incidence offers a possibility of a "light-pipe" to increase the effective solid angle that the scatterer subtends at the source. Within the limits set by the small angle of total reflection, this pipe need not be optically straight. ${ }^{4}$

The fixed baseline used for an experiment of this type reduces unwanted Doppler shifts to only those resulting from thermal, seismic, or simi- lar disturbances. To equal the predicted gravitational shift the fractional change required in the height difference is $3.27 \times 10^{-8}$ per second. Perturbing effects must be kept well below this value but this is also true for the other methods of measuring the red shift. Relative motion could be separated from the red shift by simultaneous observations of beams traveling in both directions.

${ }^{1}$ R. L. Mössbauer, Z. Physik 151, 124 (1958); Naturwissenschaften $\underline{45}, 538$ (1958); Z. Naturforsch. 14a, 211 (1959).

${ }^{2}$ Craig, Dash, McGuire, Nagle, and Reiswig, Phys. Rev. Letters $\underline{3}, 221$ (1959).

${ }^{3}$ Lee, Meyer-Schutzmeister, Schiffer, and Vincent, Phys. Rev. Letters $\underline{3}, 223$ (1959).

${ }^{4}$ We wish to thank E. M. Purcell for this suggestion.

E R R A T U M

DISLOCATION PINNING IN $n$-TYPE GERMANIUM. R. L. Cummerow and A. R. Cherry [Phys. Rev. Letters 3, 367 (1959)].

Because it was thought that the diffusion coefficient of As in Ge is much smaller than it is, the experimental results presented in this Letter were interpreted in terms of Mott pinning (pinning of dislocations dragged through a field of immobile impurity atoms). This interpretation is valid for samples deformed in a few seconds time at $500^{\circ} \mathrm{C}$, but at the annealing temperatures used $\left(600^{\circ} \mathrm{C}\right.$ and above), the more familiar Cottrell pinning (mobile impurity atom migrating to a fixed dislocation) is operating. 